\title{
OVERFLOW Validation for Predicting Plume Impingement of Underexpanded Axisymmetric Jets onto Angled Flat Plates
}

\author{
Henry C. Lee* \\ ELORET Corp., Moffett Field, CA 94035
}

\author{
Goetz Klopfer ${ }^{\dagger}$ \\ NASA Ames Research Center, Moffett Field, CA 94035
}

This report documents how OVERFLOW, a computational fluid dynamics code, predicts plume impingement of underexpanded axisymmetric jets onto both perpendicular and inclined flat plates. The effects of the plume impinging on a range of plate inclinations varying from $90^{\circ}$ to $30^{\circ}$ are investigated and compared to the experimental results in Reference 1 and 2. The flow fields are extremely complex due to the interaction between the shock waves from the free jet and those deflected by the plate. Additionally, complex mixing effects create very intricate structures in the flow. The experimental data is very limited, so these validation studies will focus only on cold plume impingement on flat and inclined plates ${ }^{1,2}$. This validation study will help quantify the error in the OVERFLOW simulation when applied to stage separation senarios.

\section{Nomenclature}

$\begin{array}{ll}D_{N} & \text { nozzle exit diameter } \\ R_{N} & \text { nozzle exit radius } \\ D_{N O} & \text { nozzle exit outside diameter } \\ D_{T} & \text { throat diameter of nozzle } \\ \theta & \text { plate angle, as measured from the nozzle centerline } \\ Z_{N P} & \text { plate distance measured from nozzle exit, measured in nozzle exit diameters } \\ M_{r e f} & \text { reference Mach number, taken to be the design nozzle exit Mach number } \\ P_{\infty} & \text { freestream pressure } \\ P_{e} & \text { pressure at nozzle exit } \\ T_{\infty} & \text { free-stream temperature } \\ \gamma & \text { specific heat ratio } \\ P r & \text { exit pressure ratio, defined as } P_{e} / P_{\infty} \\ \operatorname{Re} e_{g u} & \text { Reynold's number per grid unit length } \\ r & \text { Arc-length measured from center of plate along radius } \\ \text { CFD } & \text { computational fluid dynamics } \\ \text { OVERFLOW } & \text { Navier-Stokes computational fluid dynamics solver }\end{array}$

\section{Introduction}

$\bigcap^{\mathrm{N}}$ the Ares-I rocket, various rocket motors are used for separating the upper stage from the first stage. Due to their size and proximity to the rocket, the plumes often impinge onto the surface of the rocket. OVERFLOW, a Navier-Stokes computational fluid dynamics (CFD) solver, can be used to simulate these complex flows. While performing numerical solutions can be slow, it is still much cheaper than wind tunnel tests. Additionally, numerical simulations allow for quicker design iterations and configurations to be

\footnotetext{
${ }^{*}$ Aerospace Engineer, Advanced Supercomputing Division, MS 258-2; Henry.C.Lee@nasa.gov.
}

$\dagger$ †erospace Engineer, Advanced Supercomputing Division, MS 258-2; Goetz.H.Klopfer@nasa.gov. 
explored. However, the accuracy of the solutions needs to be quantified. Here experimental data from Reference 1 and 2 are compared with the solutions generated by OVERFLOW, to validate the predicted results. This problem was also explored using Loci-Chem, another CFD code, in reference 3 to validate that code's ability to predict plume impingement.

In this study, the experiment carried out in References 1 and 2 are simulated. The cases where the flow is shown to be considerably unsteady are simulated, to quantifying the uncertainty present in CFD simulation.

\section{Overview}

The goal of the present work is to run simulations of the plume impinging onto a plate at various angles, and see how it compares with the experiment. The inflow conditions in the chamber need to be established first, and that is performed by exhausting the plume into free air. Once the chamber conditions are determined, the impingement cases across the various plate inclination angles can be simulated. Since the flows are unsteady, time-averaged solutions are used to compare with experimental data. ${ }^{1,2}$

\section{OVERFLOW Simulation}

OVERFLOW 2.2-theta ${ }^{(4,5)}$ is used for the CFD simulations. The SST turbulence model, a two-equation model, is used to model the turbulent characteristics in the flow. ${ }^{6}$ The various corrections available in the SST model were left off. Full multigrid and multigrid cycles are used to initialize the solution, develop the plume, and help accelerate convergence. The Beam-Warming block tridiagonal scheme is used to drive the solution, and the residual is computed using central difference with scalar dissipation. The simulations are run using a combination of steady state stepping and dual time-stepping after the initial start up sequence, due to the unsteadiness of the flow.

The inflow conditions were calculated in a similar manner performed by Ref 2 . Since the experiment setup as described in Ref. 2 is vague, sea level conditions $\left(\gamma=1.4, T_{\infty}=518.67^{\circ} R, P_{\infty}=1 \mathrm{~atm}\right)$ are assumed. The Reynold's number is based on these conditions, and the reference speed is taken to be the design lip mach number of 2.2. Because the grid unit is given in millimeters, the Reynold's number per grid unit length is $R e_{g u}=51251.8$. The chamber temperature is held fixed at room temperature, while the chamber pressure is iterated until the mach disk and shocks inside the plume matched the experiment.

Grid adaption is utilized throughout the simulation. The second undivided difference of the $\mathrm{Q}$ variables along with pressure is used to determine whether a region should be refined or coarsened. Typically, the simulation is run first with full multigrid cycles, to help initialize and develop the plume, and then run with dual time-stepping to refine the the solution. The simulation switches over to steady state stepping when grid adaption is turned on. Typically in one adaption cycle, the code will mark areas for refinement/derefinement, update the grid, and then run for a short time to resolve some of the features. Several adaption cycles occur, before switching back to dual time-stepping. This process is repeated twice, before any solution averaging takes place.

\section{Geometry}

The geometry and experimental setup described in Ref. 2 is vague, so some assumptions had to be made. The nozzle is a converging-diverging type. It's shape consists of a circular arc with a radius of 21.4 $\mathrm{mm}$, equal to the throat diameter connected to a conical exit section of $15^{\circ}$ semi-angle, resulting in the exit diameter, $D_{N}$, of $30 \mathrm{~mm}$. The outside diameter, $D_{N O}$ is $40 \mathrm{~mm}$. The converging section is modeled as a circular arc with a radius of 2.56 times the throat diameter. The plate in this simulation has a width and length of $300 \mathrm{~mm}$ (10 times the nozzle exit diameter) to match the experiment. The nozzle plate separation distance in this simulation is held fixed at a distance of 2 nozzle diameters $Z_{N P}=2 D_{N}$, which is measured along the axis of the nozzle exit plane to the center of the plate. The inclination angle $\theta$ is measured from the centerline of the nozzle to the plate. The circular plate used in the experiment was changed to a square plate to allow for easier domain construction. Figure 1 shows the nozzle and plate geometry. 


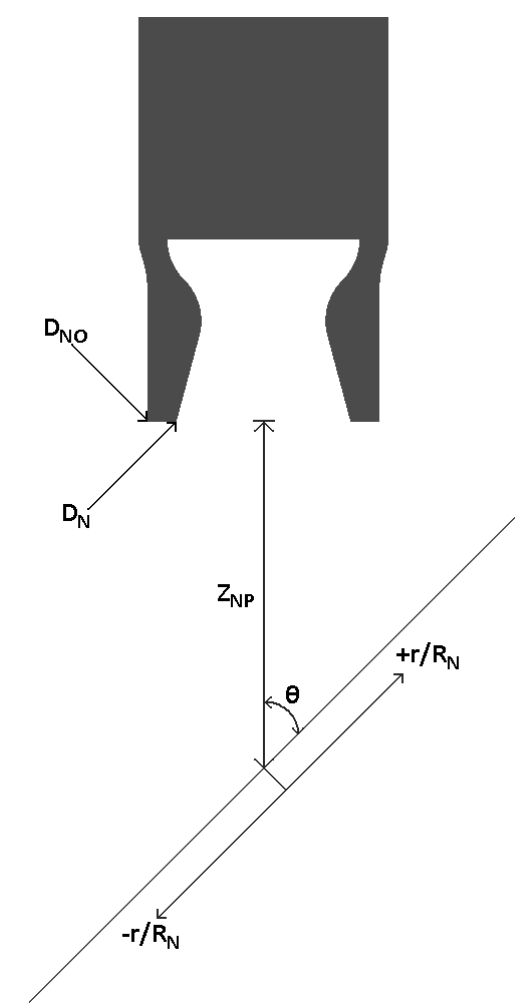

Figure 1: Geometry of Problem

A series of overset grids are used to model this problem. The grids consist of a set of near and off-body grids generated using Chimera Grid Tools version 2.2. ${ }^{7}$ Two separate grid systems are generated, one for the plume free air cases and the other for the plume impingement cases. Grid adaption is utilized in all of the cases to help resolve features in the flow field. All of the grids are three-dimensional, to ensure that any three dimensional effects of the plume are captured. Half and axisymmetric grids, after a brief study, do not capture all of the physics present in the flow.

The plume free air cases utilized a simple nozzle grid system, coupled with a series of off-body grids to capture and resolve the plume. The nozzle grid system consists of one annular grid modeling the nozzle inside and outside, and a curvilinear Cartesian core grid to remove the polar singularity. The nozzle grid and core grid make up 5.2 million grid points. The off-body grid system surrounds most of the nozzle, except for the nozzle back side, in which it is constrained to the same plane as the nozzle inflow plane. The initial off body grid system is 0.5 million grid points. After the adaption cycles, the resulting grid grew to 23.2 million grid points. Figure 2 shows the resulting grid after refinement. 


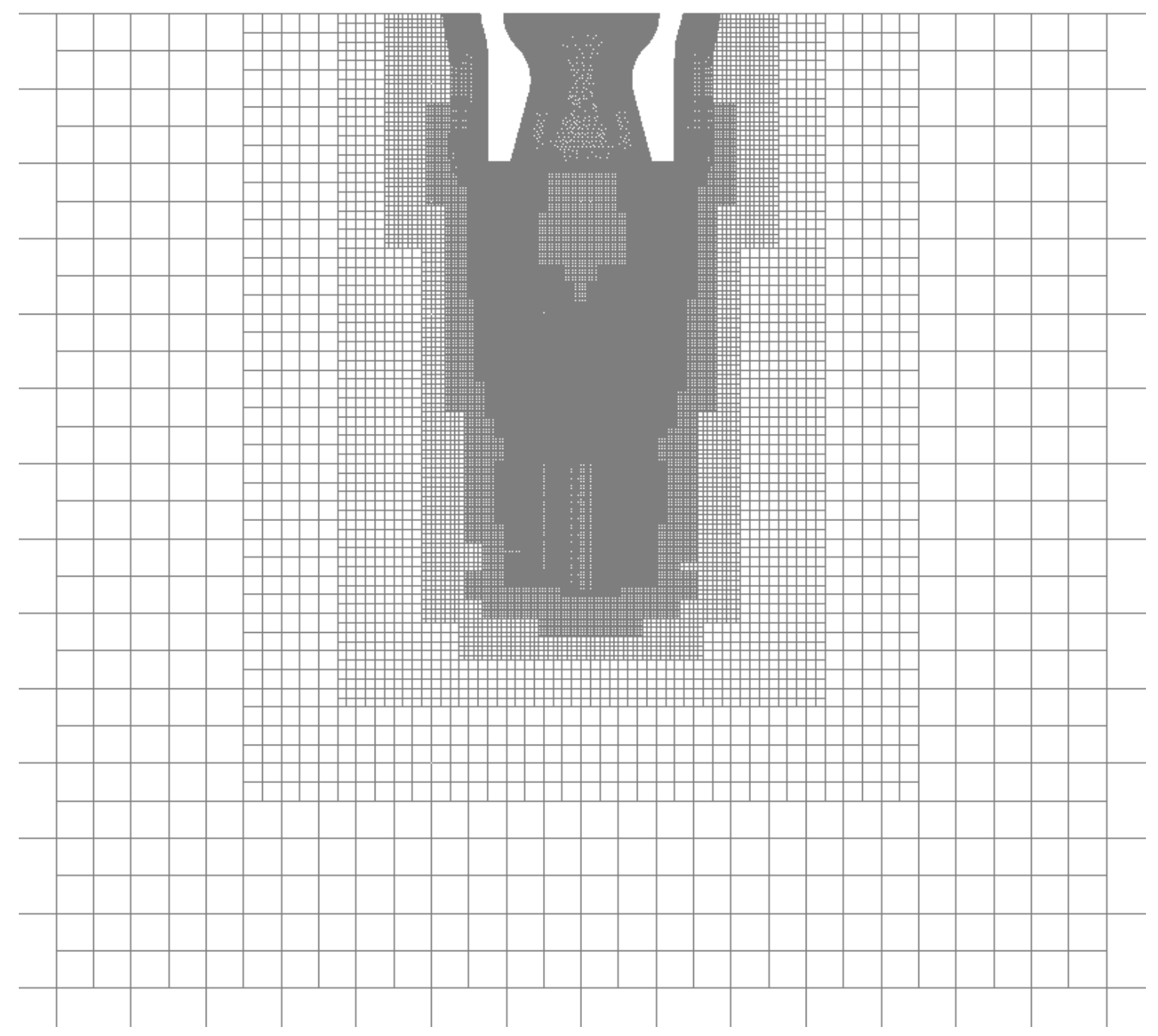

Figure 2: Plume Free Air Grid with Grid Adaption

For the plume impingement cases, the near body grids consist of four grids, which include three modeling the nozzle geometry and one modeling the plate. The nozzle grids consist of one annular grid modeling the nozzle inside and outside, and two Cartesian grids which cover the inside of the nozzle and the top of the nozzle on the outside. The core grid removes the polar singularity present in annular grids. Collectively the nozzle grids make up 5.8 million grid points. This nozzle grid is very similar to the nozzle used in the plume free air case. The difference between the two is the outside of the nozzle is extended and closed off, to form a sealed body. The plate grid is a Cartesian box grid. The points are clustered in the center of the grid, and stretch away at a distance of 3 nozzle radii measured from the center of the plate. The plate grid is 14.3 million grid points. The nozzle is rotated to the desired impingement angle, and the plate is translated to maintain the the desired separation distance. The off-body grid generation is done automatically using Chimera Grid Tools and OVERFLOW's xrays. The off-body grids completely enclose the nozzle grid, and cover most of the plate grid excluding the back side of the plate. The initial off-body grid system depending on the angle, adds anywhere from 10 to 16 million grid points. The grid adaption is limited to the area inside of 5 nozzle radii of the nozzle exit, extending down to the plate surface. The resulting grid size after adaption ranges from 94 million to 200 million grid points. Figure 3 shows the resulting grid after refinement for the $\theta=90^{\circ}$ case. 


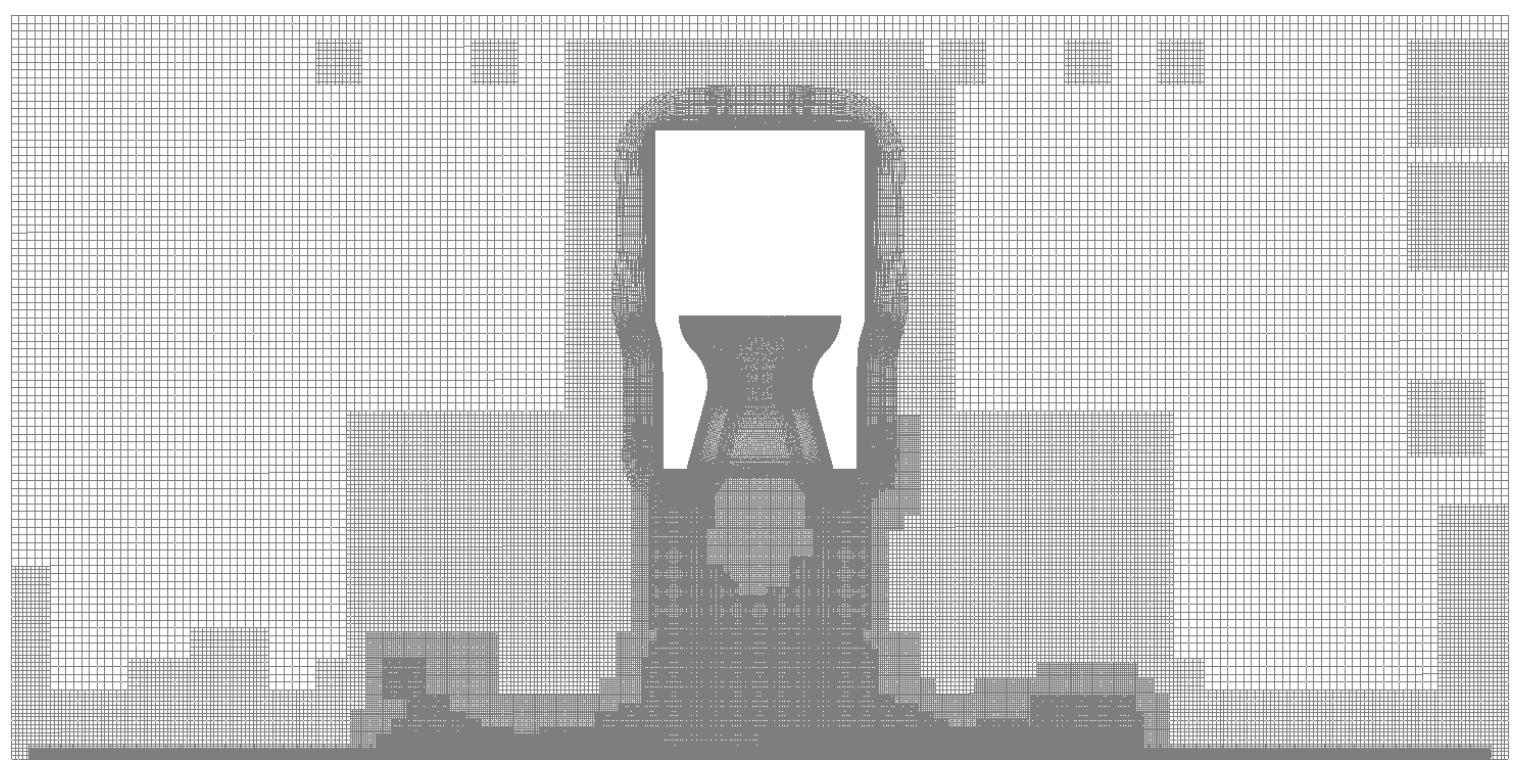

Figure 3: Example of a grid system after grid adaption $\left(\theta=90^{\circ}\right)$

\section{Inflow Determination}

The inflow is determined by iterating for the chamber pressure that compared best with experimental data. The experiment determined their inflow conditions by comparing shadowgraphs of the free jet with a program that predicted the jet shock and first mach disk location. The program was based off of a nonhomentropic method of characteristics, and also inserted exact Rankine-Hugoniot relations as the inward characteristics intersected. The chamber conditions in the experiment were then varied until the jet shock and first mach disk were in agreement with the program's solution for the specified pressure ratio. ${ }^{1,2}$

In the simulation, a sweep of pressure ratios are used to help determine the corresponding pressure ratio. The freestream pressure was set equal to sea level conditions, and the exit mach number was held equal to the design lip mach number. With those values, the area ratio between the exit and throat, the chamber pressure was backed out using one dimensional analysis. The chamber temperature was held to sea level temperature of $518.67^{\circ} \mathrm{R}$. Grid adaption was utilized, so that the features in the plume would be captured better. The pressure ratios were iterated until the mach disk and jet shock compared well with the experiment's results. The pressure ratio that compares best with the experiment is $P R=1.65$, which is $37.5 \%$ higher than the one-dimensional value. Figure 4 is a plot of the simulation's density gradient, with the experiment's shadowgraph sketch overlaid on top. 


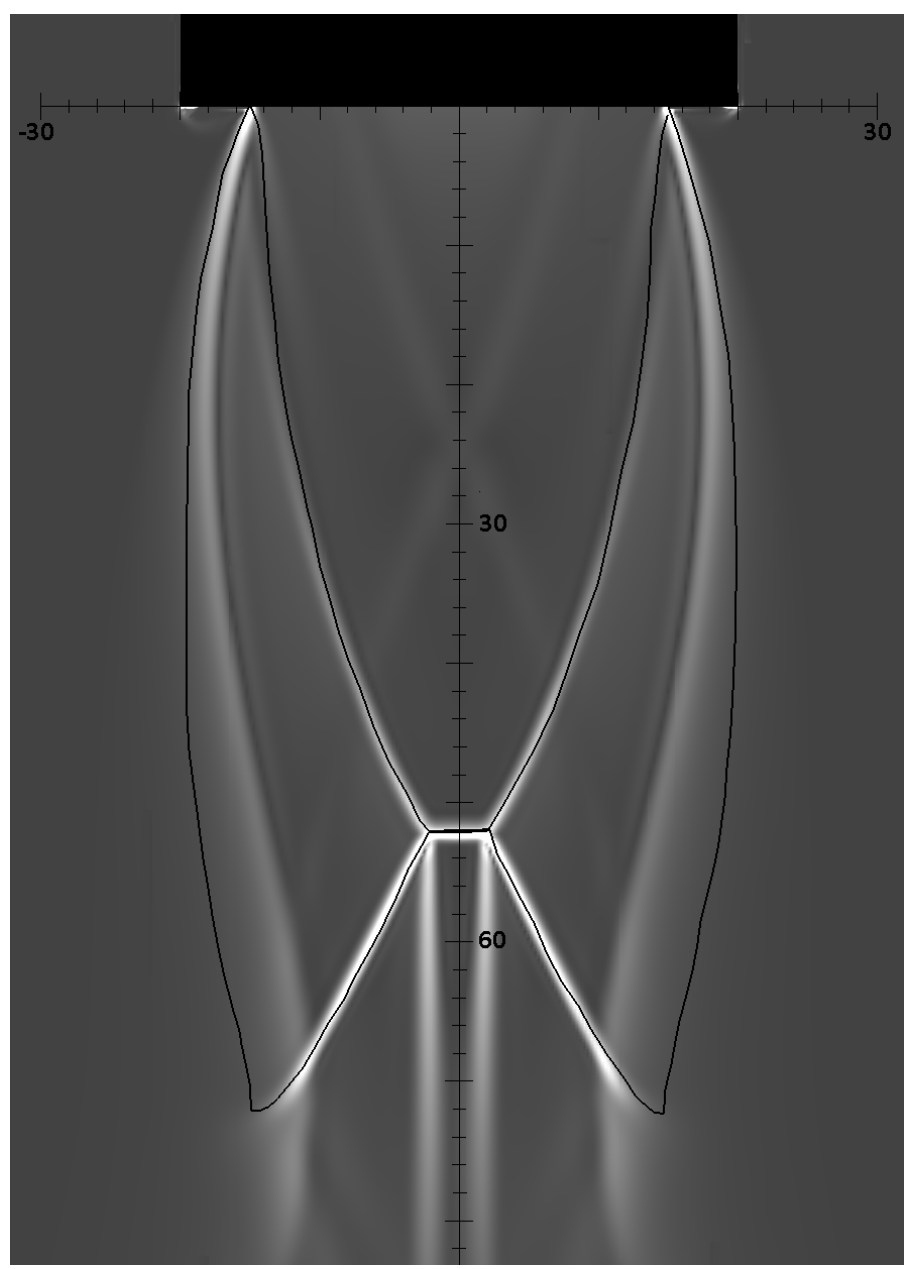

Figure 4: Plume Free Air Density Gradient Magnitude, Compared with Experiment

\section{Plume Impingement}

With the correct chamber conditions determined, the various plate angles are simulated. Due to the unsteadiness present in the flow, the solution is time-averaged using the running average option implemented in OVERFLOW. The averaging is initiated only after the initial transients in the solution have been dampened. Figure 5 show plots of pressure on the plane of symmetry at various plate inclination angles. The surface pressure is non-dimensionalized by the chamber pressure, and the arc length is non-dimensionalized by the nozzle radius. Figures 9 through 13 in the appendix show density gradient magnitude for the various plate angles. 


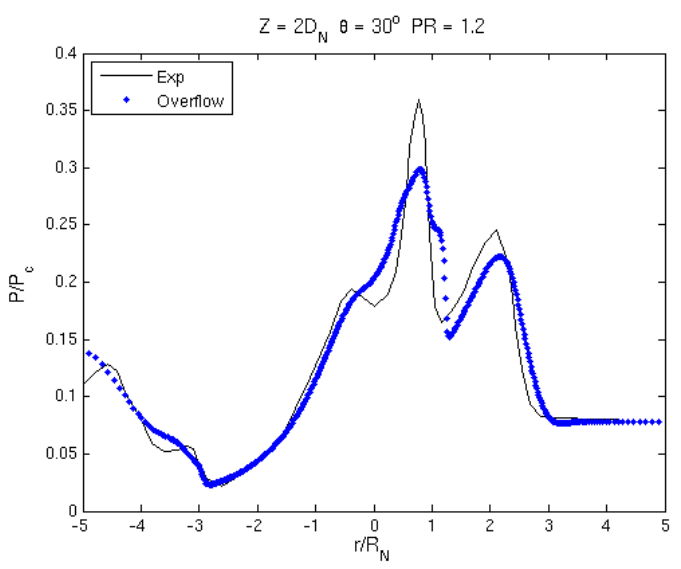

(a) $\theta=30^{\circ}$

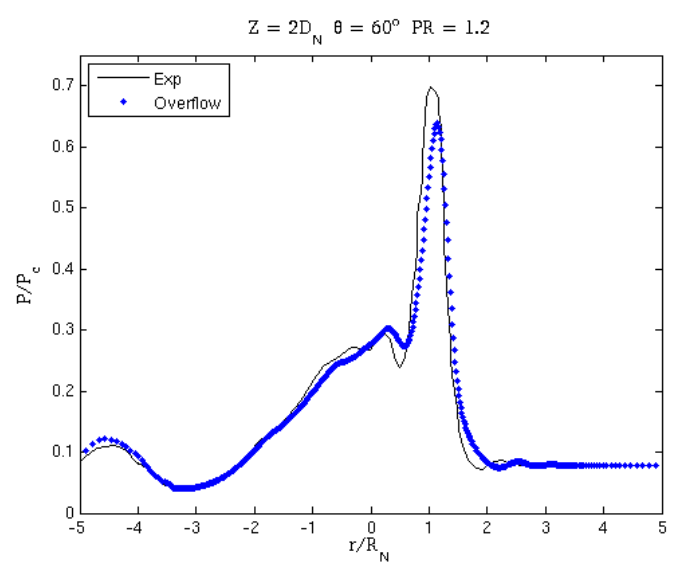

(c) $\theta=60^{\circ}$

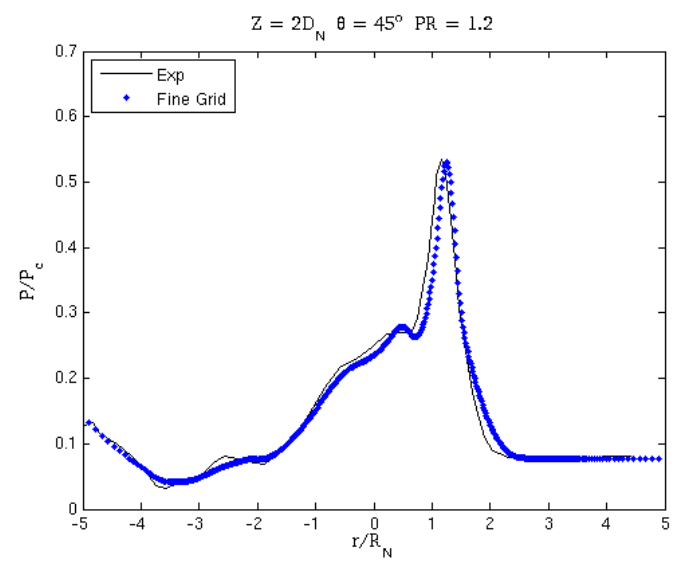

(b) $\theta=45^{\circ}$

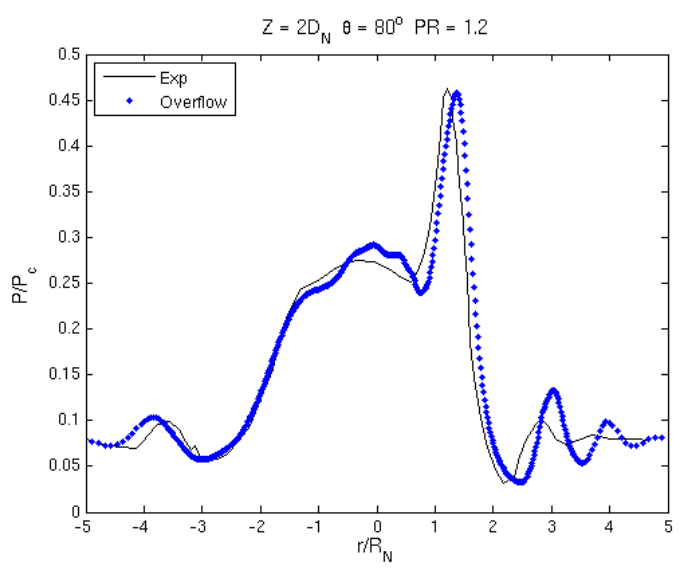

(d) $\theta=80^{\circ}$

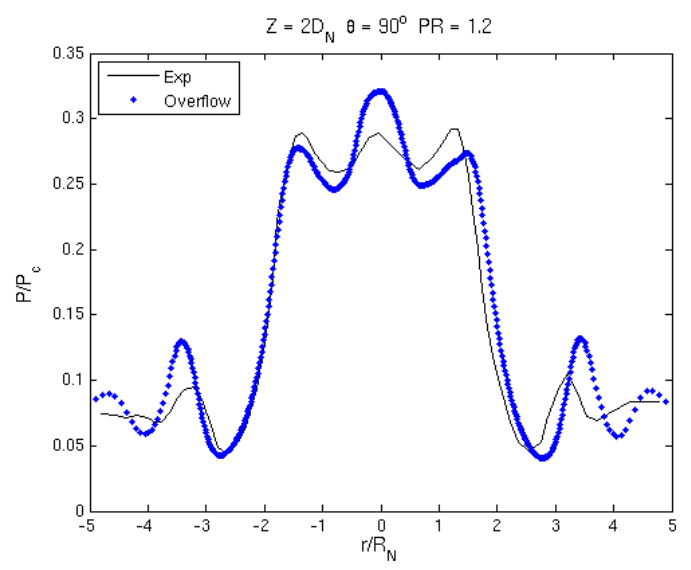

(e) $\theta=90^{\circ}$

Figure 5: Surface Pressures on the Plane of Symmetry for $Z_{N P}=2 D_{N}, P R=1.2$

The simulation results do agree fairly well with the experiment. The $\theta=30^{\circ}$ does not quite capture the peak exactly, but that might be due to insufficient grid resolution on the plate itself. The $\theta=45^{\circ}$ matches the experiment almost exactly, while $\theta=60^{\circ}$ seems to undershoot the peak. The more unsteady cases of $\theta=80^{\circ}$ and $\theta=90^{\circ}$ tend to over predict some of the secondary peaks, but the overall profile is close. The cases closer to a normal impingement angle are highly unsteady because the plate distance is very close to 
the plate, which allows for the waves reflecting from the plate to interfer with the nozzle flow. The $\mathrm{y}^{+}$on these solutions range from 1-10, with the higher values coinciding with where the pressure peaks.

To provide insight into the uncertainty present in the plume impingement CFD simulations, the forces and moments are calculated by integrating the surface pressures along the symmetry plane. The pressures are integrated using discrete trapezoidal integration from -4 to $4 r / R_{n}$. Tables 1 and 2 show the simulation's integrated forces and moments per unit length on the plate compared with the experimental values. Figure 6 show line plots of the force and moments per unit length for the simulation and experiment. ${ }^{1,2}$

\section{Table 1: Forces per Unit Length on Plate}

\begin{tabular}{ccc}
\hline \hline & & \\
Angle $(\theta)$ & Experiment & OVERFLOW \\
\hline $30^{\circ}$ & 1.02502 & 1.03520 \\
$45^{\circ}$ & 1.19249 & 1.17606 \\
$60^{\circ}$ & 1.32244 & 1.28736 \\
$80^{\circ}$ & 1.32573 & 1.35839 \\
$90^{\circ}$ & 1.31530 & 1.36393 \\
\hline \hline
\end{tabular}

Table 2: Moments per Unit Length about Centerline on Plate

\begin{tabular}{ccc}
\hline \hline & & \\
Angle $(\theta)$ & Experiment & OVERFLOW \\
\hline $30^{\circ}$ & 0.61571 & 0.60349 \\
$45^{\circ}$ & 0.47777 & 0.48456 \\
$60^{\circ}$ & 0.34923 & 0.36881 \\
$80^{\circ}$ & 0.04906 & 0.06914 \\
$90^{\circ}$ & -0.03077 & -0.01455 \\
\hline \hline
\end{tabular}

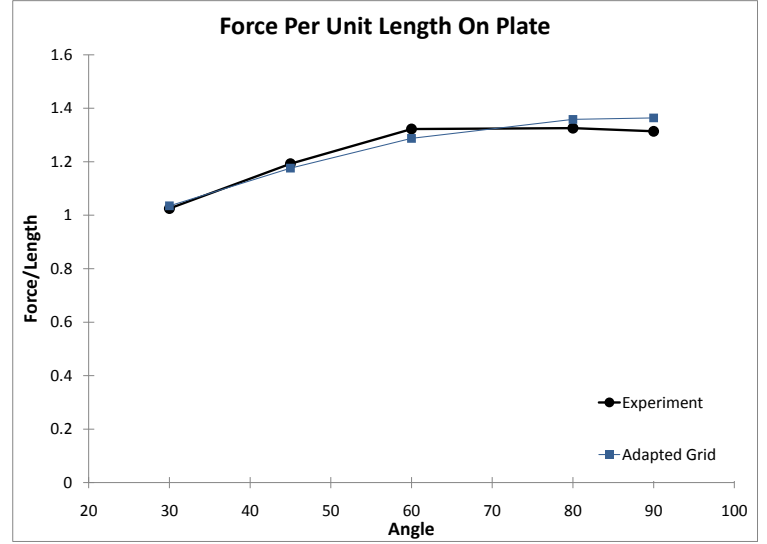

(a) Forces per Unit Length on Plate

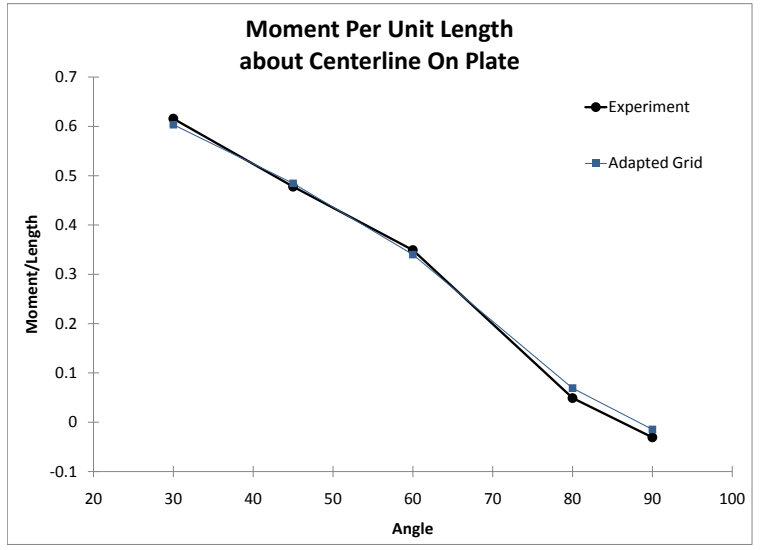

(b) Moments per Unit Length about Centerline on Plate

Figure 6: Comparison of Force and Moments of CFD and Experiment

The forces and moments per unit length in the CFD simulation compare very well with the experimental results. The force error is on the order of $1 \%$ to $4 \%$. The moment error is a little harder to quantify, because of the location of the moment reference point. The error is on the order of $1 \%$ to $4 \%$ for most of the runs, except for the near perpendicular impingement cases. The error there seems large because the values are 
closer to zero. When the moments are taken about a different point, the error is much smaller. Table 3 shows the moments taken about $r / R_{N}=-4$ for comparison.

Table 3: Moments per Unit Length about $r / R_{N}=-4$ on Plate

\begin{tabular}{ccc}
\hline \hline & & \\
Angle $(\theta)$ & Experiment & OVERFLOW \\
\hline $30^{\circ}$ & 4.7158 & 4.7443 \\
$45^{\circ}$ & 5.2477 & 5.1888 \\
$60^{\circ}$ & 5.6541 & 5.5182 \\
$80^{\circ}$ & 5.3520 & 5.5027 \\
$90^{\circ}$ & 5.2242 & 5.4412 \\
\hline \hline
\end{tabular}

\section{Time Accuracy Convergence}

The time accuracy of the solutions is also analyzed to check its sensitivity to different time-accuracy parameters. The previously computed solution is restarted and marched forward the same amount of time, with varying time-steps and sub-iterations. The instantaneous solutions are then compared against the original parameters. The surface pressures on the symmetry plane are plotted below in figure 7 and the corresponding forces and moments for each of the cases are in table 4.

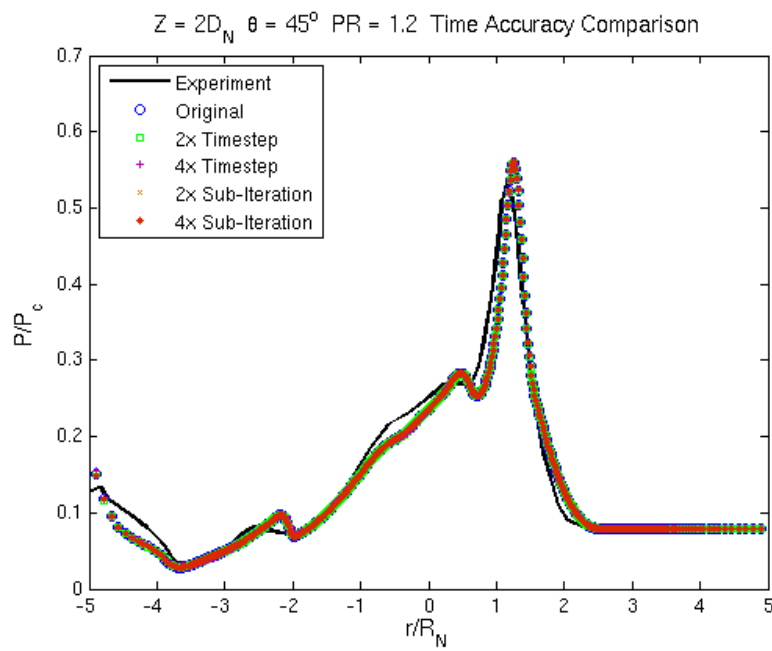

Figure 7: Pressure on Plate, Time Accuracy Comparison $Z_{N P}=2 D_{N}, \theta=45^{\circ}$

Table 4: Time Accuracy Comparison $Z_{N P}=2 D_{N}, \theta=45^{\circ}$

\begin{tabular}{lccccc}
\hline \hline & & & & & \\
Time Parameters & Original & 2x Time-step & 4 x Time-step & 2x Sub-iteration & 4 x Sub-iteration \\
\hline Convergence & 1.5 & 1.25 & 1.0 & 2.0 & 3.0 \\
Force & 1.1684 & 1.1680 & 1.1675 & 1.1685 & 1.1685 \\
Force \% Error & & $0.036 \%$ & $0.079 \%$ & $0.008 \%$ & $0.008 \%$ \\
Moment & 0.5085 & 0.5092 & 0.5096 & 0.5084 & 0.5084 \\
Moment\% Error & & $0.136 \%$ & $0.217 \%$ & $0.024 \%$ & $0.029 \%$ \\
\hline \hline
\end{tabular}


The original sub-iteration residual convergence is around 1.5 orders of magnitude, while the $2 \mathrm{x}$ and $4 \mathrm{x}$ was around 1.25 to 1 order drop. Increasing the number of sub-iterations by $2 x$ resulted in a residual subiteration convergence of 2 orders, while a $4 \mathrm{x}$ increase in sub-iterations increased the residual drop to about 3 orders of magnitude. The difference in the solution was minimal for all the cases, as seen by the plot and percent errors. This leads to the conclusion that the solution sub-iterations are reasonably converged, and the time-step is small enough to resolve most of the flow physics occurring.

\section{Grid Convergence}

The grid resolution of the problem, especially in between the nozzle and plate region, affects the solution greatly. If the cells in between the two grids are too coarse, the solution becomes too diffused, and the intensity of the pressure peaks drops off. Three grid levels are analyzed all at the same plate angle of $\theta=45^{\circ}$. The nozzle and plate grids (which total around 20-21 million grid points), are held constant for these simulations, while the off-body grid spacing is coarsened. Grid adaption is still utilized, but the level of refinement is restricted to be $2 \mathrm{x}$ and $4 \mathrm{x}$ coarser than the original fine grid computed in the previously mentioned runs. The effect of the spacing on the plate both in flow tangent's direction and the wall normal spacing is not analyzed, to limit the number of variables present in the simulation. The plate grid wallnormal spacings were held constant, to determine the effect of the off-body grids, or the plume grids on the plate. Table 5 shows the breakdown of the grid information, along with the resulting force and moment per unit length computations. Figure 8 shows the pressure distribution of the various grid levels. Figure 14 in the appendix shows the density gradient magnitude for the medium and coarse grids, which should be compared to the fine grid result in figure 10.

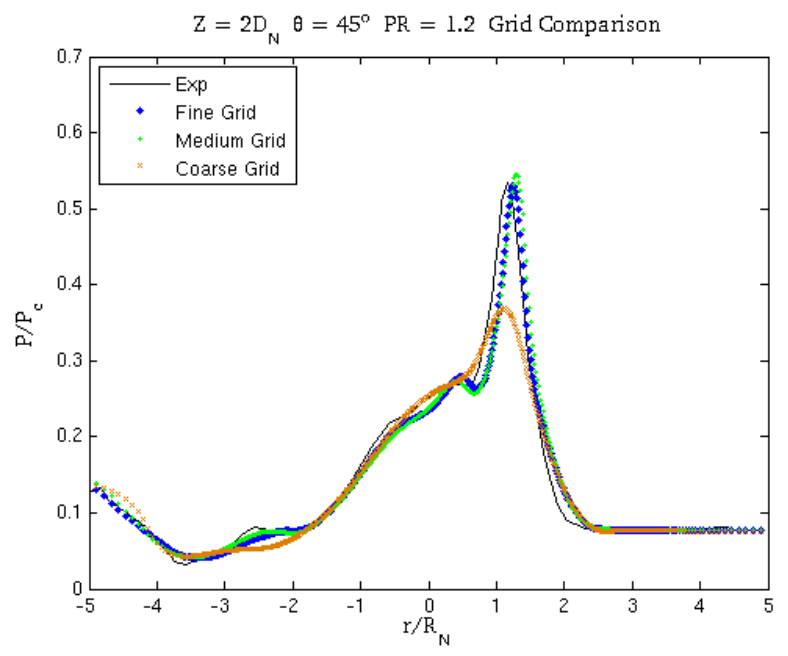

Figure 8: Pressure on Plate, Grid Comparison $Z_{N P}=2 D_{N}, \theta=45^{\circ}$

Table 5: Grid Comparison $Z_{N P}=2 D_{N}, \theta=45^{\circ}$

\begin{tabular}{lccc}
\hline \hline & & & \\
Grid Comparison & Fine Adapted Grid & Medium Adapted Grid & Coarse Adapted Grid \\
\hline \# Grid Points (Millions) & 180 & 43 & 27 \\
\# Zones & 3877 & 877 & 71 \\
Force/Length & 1.17606 & 1.1887 & 1.1336 \\
Force \% Error & $1.38 \%$ & $0.32 \%$ & $4.94 \%$ \\
Moment/Length & 0.4846 & 0.5036 & 0.4453 \\
Moment \% Error & $1.42 \%$ & $5.41 \%$ & $6.80 \%$ \\
\hline \hline
\end{tabular}


While figure 8 shows that the medium and fine grid solutions are really close, the moment calculation shows the fine grid is closer to the experiment (Note: The accuracy in the experimental data is still being determined). Grid independence seems to fall somewhere in between the medium and fine grid. The coarse grid fails to capture the extent of the pressure peak, but the solution does resemble the experiment's pressure profile. As evident in figure 14 the shear layers in the plume are sensitive to the effective grid resolution, and coarser grids tend to smear out the shear layersn. Grid resolution also affects the intensity of the pressure peaks, and in turn increasing the moment error.

\section{Conclusion}

OVERFLOW, along with the SST model, is able to capture the impingement pressure and therefore force and moments with reasonable accuracy. Full three-dimensional grids should be used to capture the threedimensional unsteady effects caused by the plume. Additionally, perpendicular impingements are harder to resolve accurately, in comparison to inclined plates. The accuracy of the pressure, force and moment values are dependent on the grid resolution. The finer the grid resolution between the nozzle exit and the surface impingement, the more accurate the solution will be. However, reasonable accuracy around 5-7 percent can be obtained with a coarser grid, but the details of the plume most likely not be resolved. To obtain more accurate answers, higher grid resolution is needed.

\section{Acknowledgments}

The help provided by the following is greatly appreciated: Tom Pulliam, William Chan, Shishir Pandya, James Kless, Jeff Onufer, Michael Olsen, Cetin Kiris, Robert Childs, Dennis Jespersen, Tim Sandstrom

\section{References}

\footnotetext{
${ }^{1}$ Lamont, P.J., and Hunt, B.L., "The impingement of underexpanded, axisymmetric jets on perpendicular and inclined flat plates," Journal of Fluid Mechanics, Vol. 100, Pt. 3, 1980, pp.471-511.

${ }^{2}$ Lamont, P.J., and Hunt, B.L., "The impingement of underexpanded, axisymmetric jets on normal and inclined flat plates," University of Bristol, Aero. Dept. Rep. PJL/7701/BLH/7703, Bristol, UK, 1977

${ }^{3}$ Canabal, Francisco., Mishtawy, Jason., and West, Jeff., "Loci-Chem Computational Fluid Dynamics Code Validation For Predicting Plume Impingement Pressure and Heating," Unpublished NASA MSFC Report, Huntsville, AL, Sept. 2008.

${ }^{4}$ Nichols, Robert H., and Buning, Peter G., "User's Manual for OVERFLOW 2.1," NASA Unpublished Report, Aug. 2008.

${ }^{5}$ Nichols, Robert., Tramel, Robert., and Buning, Pieter., "Solver and Turbulence Model Upgrades to OVERFLOW 2 for Unsteady and High-Speed Applications," AIAA Paper 2006-2824, 24th AIAA Applied Aerodynamics Conference, San Francisco, CA, June 2006.

${ }^{6}$ Menter, F.R., and Rumsey, C.L., "Assessment of Two-Equation Turbulence Models for Transonic Flows," AIAA Paper 94-2343, June 1994.

${ }^{7}$ Chan, William., "The OVERGRID Interface for Computational Simulations on Overset Grids," AIAA Paper 2002-3188, 32nd AIAA Fluid Dynamics Conference and Exhibit, St. Louis, MO, June 2002.
} 


\section{Appendix}

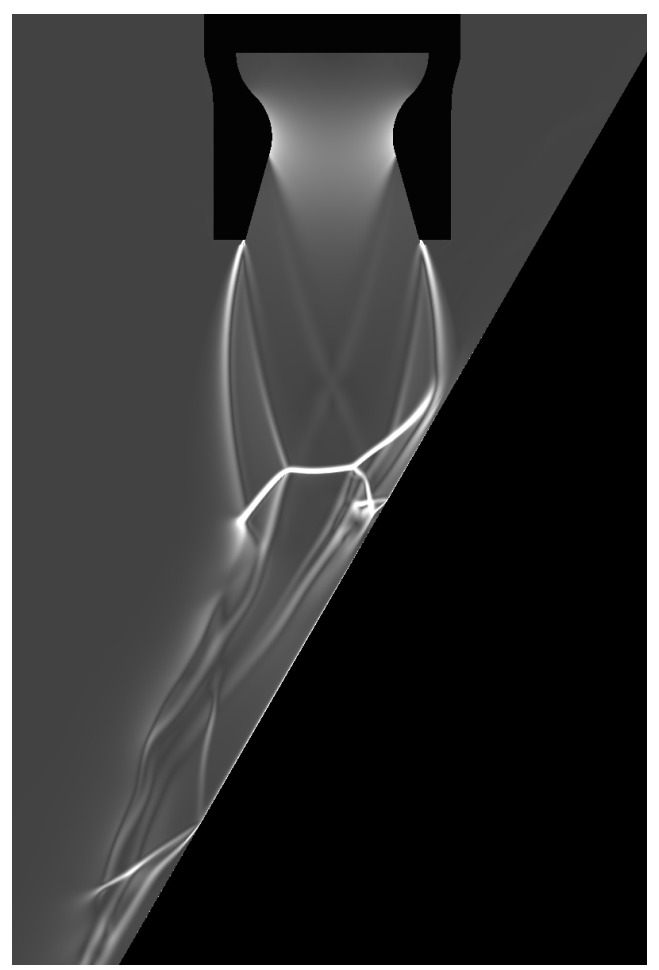

Figure 9: Density Gradient Magnitude for $Z_{N P}=2 D_{N}, P R=1.2, \theta=30^{\circ}$

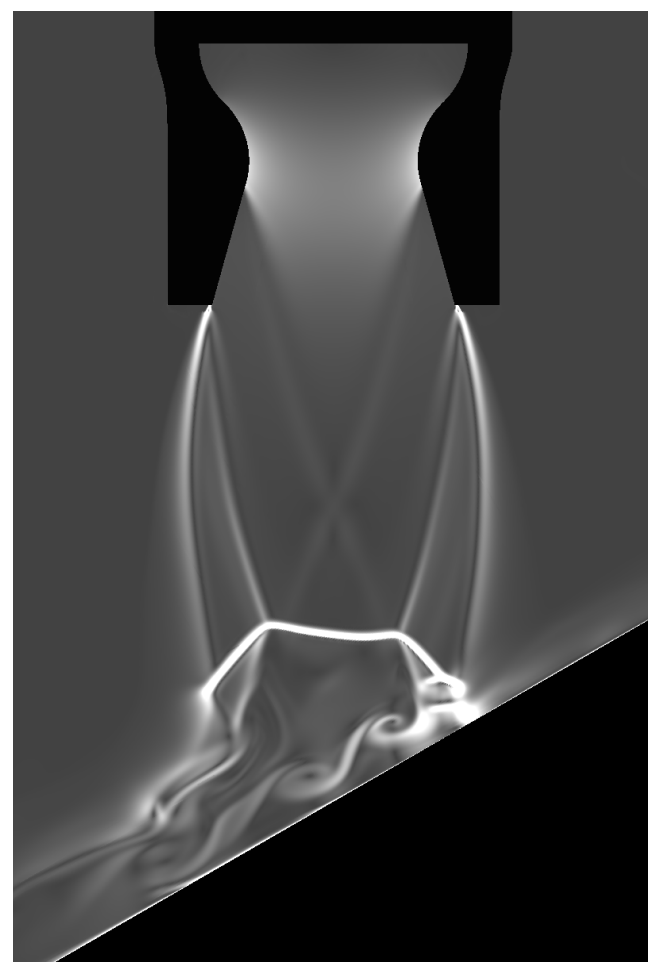

Figure 11: Density Gradient Magnitude for $Z_{N P}=2 D_{N}, P R=1.2 \theta=60^{\circ}$

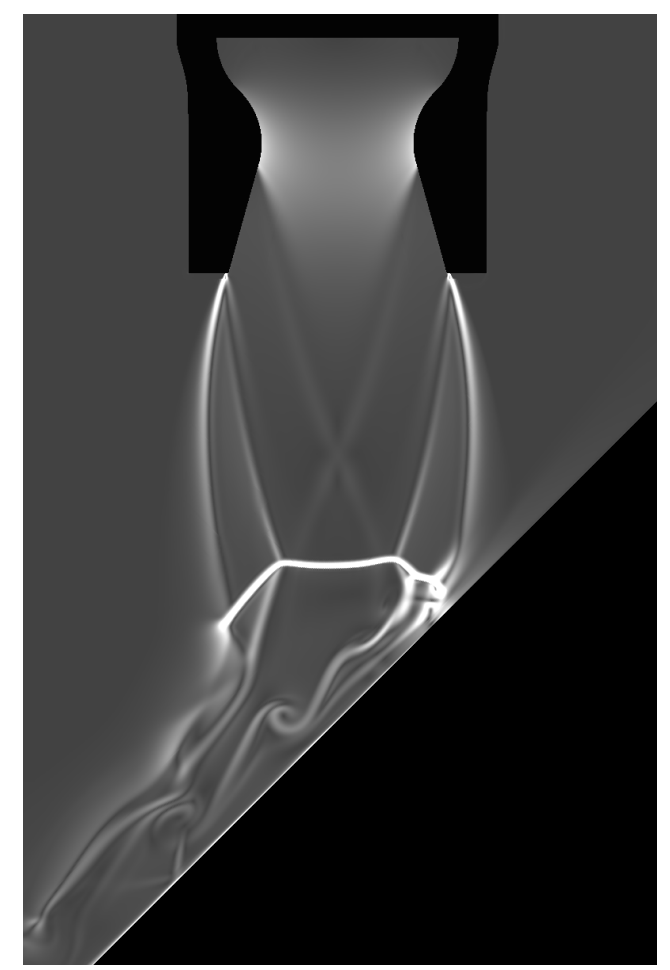

Figure 10: Density Gradient Magnitude for $Z_{N P}=2 D_{N}, P R=1.2, \theta=45^{\circ}$

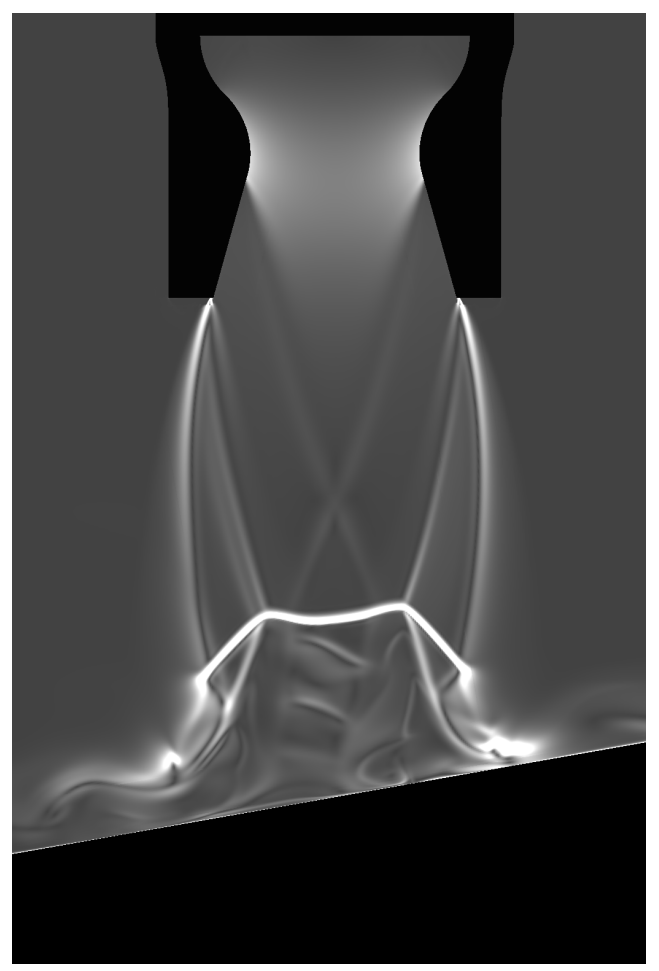

Figure 12: Density Gradient Magnitude for $Z_{N P}=2 D_{N}, P R=1.2 \theta=80^{\circ}$ 


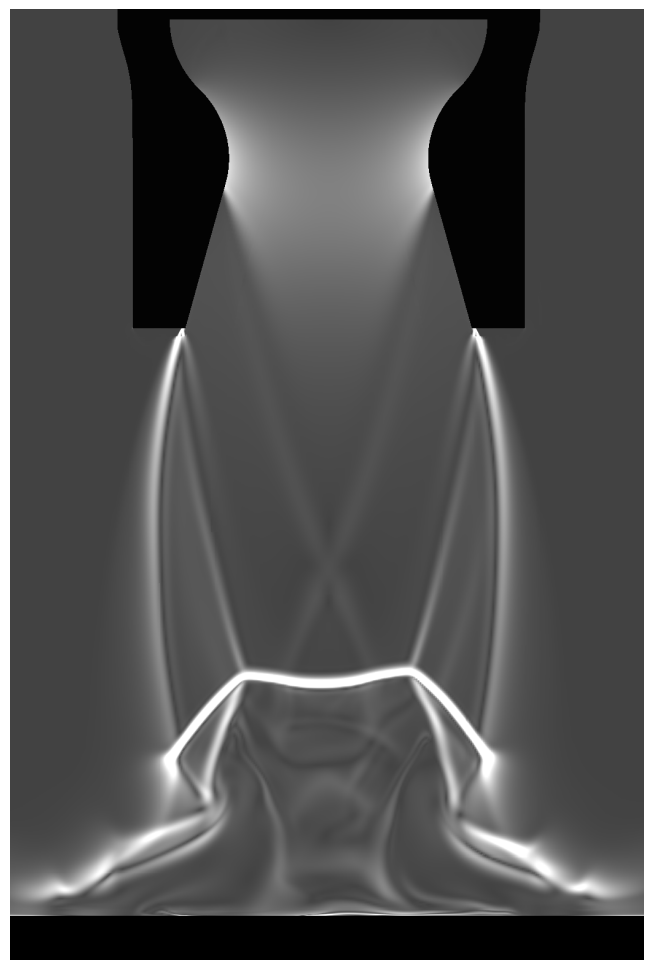

Figure 13: Density Gradient Magnitude for $Z_{N P}=2 D_{N}, P R=1.2 \theta=90^{\circ}$

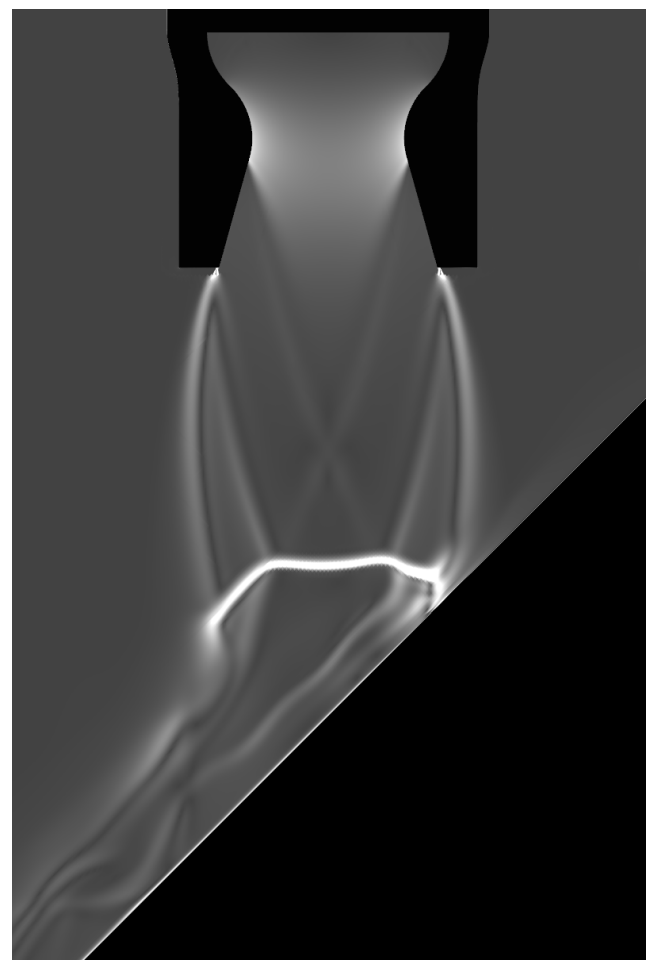

(a) Medium Grid

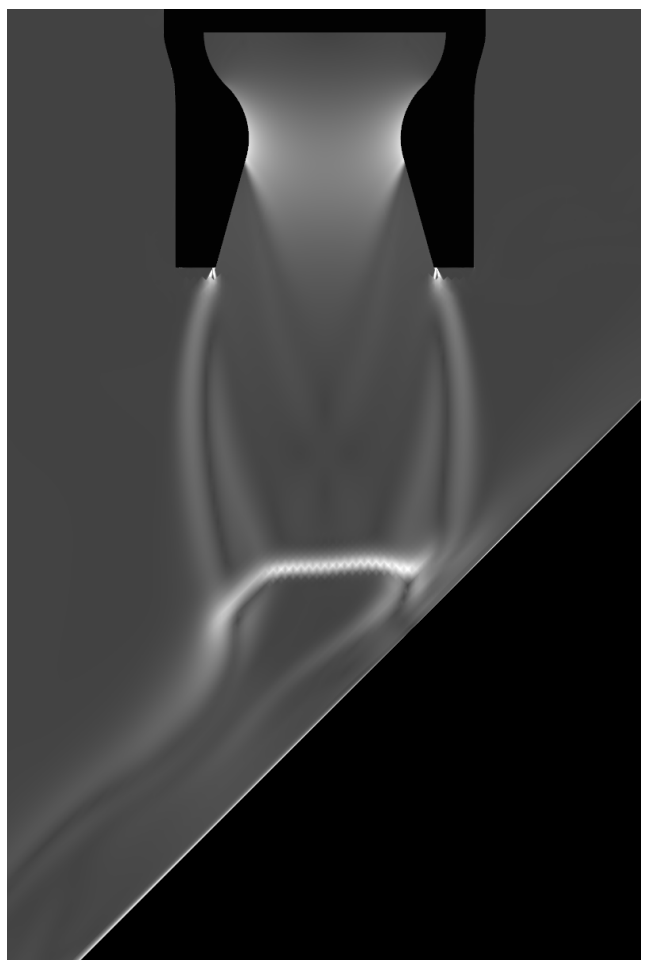

(b) Coarse Grid

Figure 14: Density Gradient Magnitude for Grid Comparison $Z_{N P}=2 D_{N}, P R=1.2$ 